\title{
Simulating the Influences of Aging and Ocular Disease on Biometric Recognition Performance
}

\author{
Halvor Borgen ${ }^{1}$, Patrick Bours ${ }^{1}$, and Stephen D. Wolthusen ${ }^{1,2}$ \\ 1 Norwegian Information Security Laboratory, Gjøvik University College, \\ P.O. Box 191, N-2802 Gjøvik, Norway \\ 2 Information Security Group, Department of Mathematics, Royal Holloway, \\ University of London, Egham Hill, Egham TW20 0EX, United Kingdom
}

\begin{abstract}
Many applications of ocular biometrics require long-term stability, yet only limited data on the effects of disease and aging on the error rates of ocular biometrics is currently available. Based on pathologies simulated using image manipulation validated by opthalmology and optometry specialists, the present paper reports on the effects that selected common ocular diseases and age-related pathologies have on the recognition performance of two widely used iris and retina recognition algorithms, finding the algorithms to be robust against many even highly visible pathologies, permitting acceptable re-enrolment intervals for most disease progressions.
\end{abstract}

\section{Introduction}

High levels of accuracy combined with being relatively difficult to forge are making ocular (iris and retina) biometrics attractive for identification and authentication in a number of areas. These include border control or other machine readable identification documents where, unlike in the case of access control to restricted areas, it is highly desirable to have long intervals between re-enrolment. While long-term stability of features such as fingerprints is well understood and limited research has also been conducted on the impact e.g. of dermal pathologies [1], this has not been the case for ocular features, particularly for the robustness of ocular biometrics to pathologies in general and the stability of the features used for recognition over time given aging and pathologies. Unlike in the case of fingerprints and other external features such as facial images, where medical and police records provide longitudinal records of feature stability and in many cases also disease progression, we are not aware of such data sets existing for iris and retina images, tracking disease and age progression from a healthy baseline and particularly using the multiple baseline images required for accurate initial enrolment of the ocular features. We have therefore chosen to acquire high-quality images directly in case of retinal images and using an existing database for iris images [2] as a baseline images for enrolment in biometric systems, simulating selected pathologies. To ensure that the simulations provided a faithful representation of these pathologies, all simulated stages were validated by opthalmology and optometry specialists.

M. Tistarelli and M.S. Nixon (Eds.): ICB 2009, LNCS 5558, pp. 857-867 2009.

(C) Springer-Verlag Berlin Heidelberg 2009 


\section{Biometric Recognition Techniques Based on Ocular Features}

For the purposes of this paper, a single algorithm and parameterization was chosen for both retina and iris recognition techniques. While different algorithms, signal acquisition mechanisms, and tuning are likely to yield different quantitative results, the main purpose of this paper is to explore whether these biometric techniques exhibit sufficient long-term stability, which is a sufficiently coarse requirement so as to allow qualitative extrapolation to other ocular biometric systems.

Retina Recognition. The suitability of the retinal vascular pattern for identification purposes was posited by Simon and Goldstein, later studies by Tower established experimentally that even identical twins exhibited randotypical retinal vascular patterns [345]. While technical solutions for capturing retinal images and their use in biometrics was already developed in the 1970s [6], it has not seen widespread adoption. A retina scanner must illuminate an annular region of the retina through the pupil either in the infrared or visible spectrum. This region centered on the fovea is approx. $10^{\circ}$ off the visual axis of the eye. For recognition, the reflected vasculature contrast is recorded, capturing the pattern of blood vessels on the retina, the choroidal vasculature, and surrounding contrast. Successful acquisition requires alignment and fixation as well as a unobstructed optical pathway between sensor and the retina. The latter implies that the optical system must be able to accommodate different focal lengths so subjects can reliably focus on the target since eyeglasses would introduce distortions. Moreover, as the remainder of this paper will argue, it is also important to have a well-characterized optical pathway in the eye itself as certain diseases and age-related changes may also affect signal acquisition. However, this pathway and dynamically adjustable illumination also provides opportunities for liveness tests (e.g. dilation, depth effects, detection of moisture). In addition, a counterfeit retina or image must not only replicate the same vascular pattern in multiple focal layers but also simulate reflectivity of the retina and optical pathway as well as the flexible lens, the focusing of incoming and reflected beams, and its dynamic changes in response to external stimuli. Early recognition mechanisms include [789]. Here we present a system based on Hill's original design adapted to readily available acquisition as described in [10].

Iris Recognition. The randotypical patterns emerging during the growth of the eye yield a large feature space and are not only distinct among indiviuals but also different for each eye of the same individual [116]. This was identified as a suitable feature for biometric identification and verification by ophthalmologists Flom and Safir [12] while Daugman developed the dominant algorithm for encoding and matching 1314. The iris is a part of the central, or uveal, coat of the eye and consists of a trabecular meshwork [5]15] of elastic connective tissue. This trabecular meshwork pattern is completed during the first eight months of gestation, and remains stable apart from possible depigmentation [16] and the 
effects of disease and trauma. Clinical evidence suggests that iris color change in adolescence has no effect on trabecular patterns while post-adolescence, depigmentation, and shrinking of the pupillary opening can occur with advanced age in healthy irises [11. An iris scan requires a high-resolution grey-scale image (approx. 200 pixels across the diameter of the iris), preferably illuminated by infrared light. Since signal acquisition typically requires the capture of multiple frames, several dynamic liveness tests can be integrated in the acquisition, e.g. tracking pupil to iris diameter ratios. The diameter of the pupil is constantly oscillating due to the complex interplay of the muscles of the iris [11, which can also be induced by changes in illumination. Similarly, the fact that the lens floats outside the body of the eye and that the iris lies beneath the cornea creates a detectable difference in geometry if a custom contact lens is used to replicate the pattern of the iris itself. Depending on the acquisition mechanism used, a segmentation algorithm is required to identify the iris region as well as to characterize images of sufficient quality that are e.g. not excessively occluded by eyelids. In addition, distortions introduced by eyelashes, the presence of specularity (e.g. owing to poor illumination characteristics) or the iris image being out of focus can also degrade the acquired signal. Moreover, changes in lighting condition (as well as other stimuli) can result in pupillary dilation, which in turn will result in a nonaffine transformation of the iris. For the purposes of this paper, the algorithm by Daugman [13] in the implementation by Masek was chosen 17 (modified only to handle a slightly different image size). This algorithm must first find both the pupillary boundary and the outer boundary of the iris, the limbus. Once the searches for both boundaries have reached the single pixel precision, a similar approach to detect curvilinear edges is used to localize the eyelid boundaries. The path of contour integration in the operator is changed from circular to arcuate to fit the statistical estimation methods with the parameters to describe optimally the available evidence for each eyelid boundary. Each of the isolated iris patterns is then demodulated to extract its phase information using quadratic 2D Gabor wavelets.

\section{Ocular Diseases and Their Simulation}

A number of diseases and age-related developments of the eye can have a negative impact on the features and characteristics relevant to iris and retina recognition; some features may affect only one technique in particular while others can affect both approaches at the same time. The following section provides a brief overview of selected common pathologies and their potential impact.

Glaucoma. 18] causes the pressure of the fluids inside the eye to rise slowly, resulting in loss of visual acuity or blindness. In some severe cases, the increase in pressure causes severe damage to the optic nerve. Two types of adult glaucoma can be distinguished, open and closed angle glaucoma. Here, canals providing drainage to the eye become clogged over time, causing the pressure in the inner eye (intraocular pressure, IOP) to slowly rise owing to insufficient drainage of fluids. Closed angle (acute or narrow angle) glaucoma, is more rare. The main 
difference is that the pressure rises very quickly due to severe clogging or blocking of the drainage canals. Here, the peripheral iris outer edges block the anterior chamber angle and the drainage canals because the pupil dilates too quickly or too much as may happen when entering a dark room. High pressure can result in a corneal edema, which in turn can create corneal scarring. Also of interest in this context is that treatment of severe narrow angle closure is accomplished by removing a segment of the outer iris edge (trabeculectomy) while less severe cases respond to medication (latanoprost), which in turn may result in pigmentation changes 19. Macular degeneration 20]21] is a (generally age-related, hence AMD) disease of the retina. The macula is the central portion of the retina responsible for fine details in the vision. Loss of vision occurs when photoreceptors in the macula degenerate. In atrophic (dry) AMD, also referred to as geographic atrophy, irregular pigmentation of the macular region occurs, but no hemorrhage or exudation is evident in the macular region. Here, yellow-white deposits accumulate in the retinal pigment epithelium (RPE) tissue beneath the macula (see 22 ). These deposits, called drusen, are waste products from the photoreceptor cells while exudative AMD is characterized by subretinal choroidal neovascularization. Cataracts are the result of a breakdown of cellular waste products in the lens, resulting in a blurred or cloudy lens. Three types can be distinguished: Nuclear, cortical, and subcapsular cataracts. Nuclear cataracts are the most common form in the center of the lens while cortical cataracts (common in diabetic patients) form in the lens cortex, gradually extending to the center of the lens. Subcapsular cataracts start at the back of the lens working its way forward and is associated with retinitis pigmentosa, diabetes, and high dosage of steroids 23. Retinopathy refers to several types of retinal diseases affecting the fine retinal vasculature. In case of hypertensive retinopathy, high blood pressure damages and causes hemorrhaging of the vasculature; this may also be accompanied by exudates and cotton-wool spots. In case of ocular arteriosclerosis, retinal arteries harden, causing local hypertension in arteries and also in the capillaries. This results in a change in the capillary structure and may also cause hemorrhages, drastically changing the blood vessel pattern. Other possible signs of the disease are exudates and macular edema 24. Diabetic retinopathy is the most common diabetic eye disease and one of the leading causes of blindness [24]. Here, the vasculature either swells, resulting in a leak of fluids, or pathological angiogenesis can ensue. Leaking vasculature results in blurry vision or, if affecting the macula, result in a macular edema. Two types of diabetic retinopathy are distinguished, proliferative and non-proliferative, the former is the more severe form. Non-proliferative retinopathy does not include vascular growth, but hemorrhages and exudates will also occur. Pathological angiogenesis is the abnormal rapid proliferation of blood vessels which is caused by cells receiving insufficient supplies of oxygen, as can e.g. be the case in tumor growth. This results in the release of angiogenic molecules that attract inflammatory and endothelial cells. The inflammatory cells secrete molecules that intensify the angiogenic process 25. While angiogenesis can occur in any location, is retinal pathological angiogenesis that is of particular interest. Keratitis refers to an inflammation of the 
cornea, which can have a number of different etiologies. Common symptoms for these are dullness of the cornea that sometimes turn into a grey-white connective tissue called macula cornea. Bacterial keratitis is caused by a bacterial infection and introduces a small grey-white collection of leukocytes (white blood cells) causing the surface of the cornea to turn dull because of the edema, with fungal keratitis yielding similar symptoms. Further variations can be caused by viral keratitis and photokeratitis [26].

\section{Disease Simulation}

Lacking a well-characterized longitudinal data series for ocular pathologies described in section 3, disease progression had to be simulated followed by subject expert validation in each case. The source images for healthy retinae and iridae were the UBIRIS database 2 in case of iridae and a data set collected by the authors in collaboration with Sykehuset Innlandet Lillehammer, Norway. For all diseases, three progression stages simulations were produced, with the first simulating disease onset and the final one advanced stages of a given disease, with the simulated images being validated by ophthalmologists.

\subsection{Diseases Affecting the Iris and Cornea}

Many pathologies, particularly those affecting the cornea, impact both iris and retina images. The following is a selection of common pathologies and symptoms likely to be encountered in aging populations and long-term biometric identification and authentication environments. The UBIRIS images are from 241 subjects and two sessions with a size of $200 \times 150$ pixels and the difference between the two series being the addition of noise in the second series. For the purposes of the present paper only the noise-less image series was used (see figure 1a for an example of an original image from this series).

Keratitis and Infiltrates. To simulate several types of keratitis, infiltrates of different sizes were spread over the corneal area. For severe cases, this will result in a whitening of the entire corneal area. Figures $1 \mathrm{~b}$ through $1 \mathrm{~d}$ illustrate different stages of central keratitis and infiltrates.

Blurring and Dulling of the Cornea. Corneal bleaching or clouding can be caused by diseases such as Glaucoma, Maroteaux-Lamy syndrome, Hurler's syndrome, KID syndrome, and a number of diseases similar to keratitis and scarring of the cornea (see fig. 1e for advanced-stage corneal bleaching).

Scarring and Surgery of the Cornea. Corneal scarring can occur in patients suffering from glaucoma or other diseases where the intraocular pressure builds up and creates corneal hemorrhaging. Corneal scarring is also common after injuries where a physical object has been in contact with the cornea. Glaucoma surgery is performed by removing a small part of the iris, at the outer edge, to release the pressure. This leaves a black mark on the iris, with unclear edges. Change 


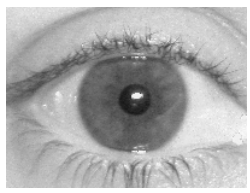

(a) Original image prior to manipulation (from [2])

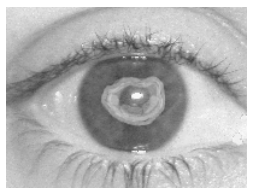

(b) Central keratitis, advanced stage simulation

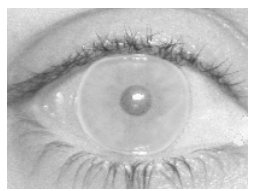

(e) Simulated corneal bleaching

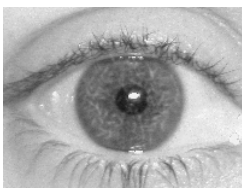

(c) Simulated infiltrates across entire cornea, low density

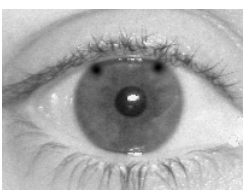

(f) Simulated corneal scars from glaucoma surgery

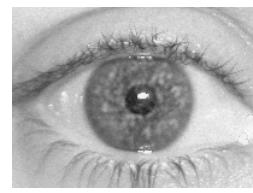

(d) Simulated infiltrates across entire cornea, high density

Fig. 1. Keratitis and infiltrates, corneal bleaching and scarring

in iris color is not normally observed, but can occur when using glaucoma medication [19. Figure 1f] shows an example of scars typical of glaucoma surgery at intermediate size.

Angiogenesis. Abnormal vascularization can occur whenever a subject suffers from vein occlusion, or lack of oxygen to the cornea. The latter is often related to wearing contact lenses but can also be caused by tumor growth.

Tumors and Melanoma. In addition to the vascular growths noted in the preceding paragraphs, deformations and larger-scale occlusions owing to hemorrhaging or melanoma are of particular interest. To simulate such pathologies, entire sections of the iris images were colored uniformly either black or white (given that hemorrhages and tumors in the iris are dark while conjunctival tumors spreading to the iris are light and pale). While tumors and hemorrhages can also affect the pupil, the simulation did not modify the pupil as this tends to affect image segmentation algorithms, not the recognition algorithms that are of interest in the present paper (see 22] for examples of a hemorrhages).

\subsection{Diseases Affecting the Retina}

Retinal images used in the experiments were acquired at Sykehuset Innlandet, Lillehammer, Norway using a standard Topcon TRC-50IX mydriatic retinal camera. The digital image sensor affixed to the TRC-50IX yielded a color (RGB) image at 768 by 576 (PAL resolution, square pixels), with the images stored in an uncompressed TIFF format under illumination by visible light provided by two integral light sources, a $100 W$ (max.) halogen lamp for observation, and a max. 300 Ws Xenon flash for photography. The camera had an angular coverage of $50^{\circ}, 35^{\circ}$, and $20^{\circ}$ and total observation magnification of $10 x$ at $50^{\circ}, 13.3 x$ at $35^{\circ}$, and $23.3 x$ at $20^{\circ}$, respectively as well as photographic magnification of 
$1.84 x$ at $50^{\circ}, 2.45 x$ at $35^{\circ}$, and $4.28 x$ at $20^{\circ}$, respectively (at zero diopter) [10]. For the symptoms described in the remainder of this section, it should be noted that these are not specific or exclusive to particular diseases, the latter are provided mainly as examples. Cataracts and Lens Blurring Cataracts can result in a blurring of the lense, which affects the ability of the lens to focus light onto the retina. Figure 2a illustrates a severe cataract that would also significantly affect a patient's vision. Hemorrhaging Retinal hemorrhaging is common in diseases involving hypertension, and in diabetes-related ocular diseases. Only hemorrhages within the recognition algorithm's scan circle were considered in the simulation. Hemorrhages can appear as both small red dots, and large collections of blood, both of which were simulated, although figure $2 \mathrm{~b}$ only shows large-area hemorrhaging.

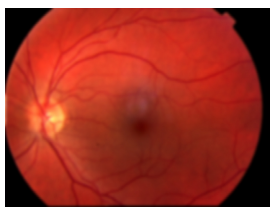

(a) Cataract

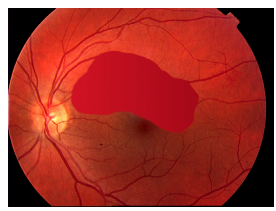

(b) Hemmorrhaging

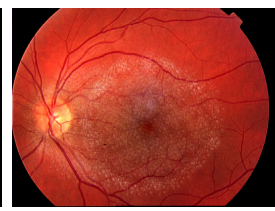

(c) Drusen

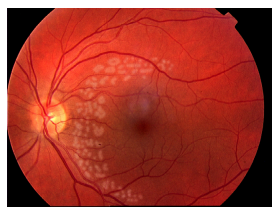

(d) Cotton-wool spots

Fig. 2. Retinal hemorrhaging, cataracts, Drusen and cotton-wool spots

Exudates and Drusen. Cellular waste materials called drusen are e.g. found in macular degeneration while exudates and cotton-wool spots are found in several different retinopathies, including hypertensive and diabetic retinopathy, macular degeneration, and arteriosclerosis. Figure 2c shows a simulation of drusen while figure $2 \mathrm{~d}$ illustrates simulated cotton-wool spots.

\section{$5 \quad$ Experimental Results}

For the iris recognition stage, 17 subjects were chosen based on a combination of the intra-class and inter-class Hamming distances. These Hamming distances indicated that for our data set, a threshold between 0.30 and 0.35 was optimal, and the threshold was set at 0.35 for matching (i.e. a Hamming distance over 0.35 between the healthy template and the simulated diseased image led to a rejection; for these experiments only the FRR was of interest). The simulations were carried out on a set of manipulated images from one healthy image, and the remaining four healthy iris images as templates in four separate simulations. Table 1a summarizes the findings for all simulations. We note that the main reason for the false acceptances observed was faulty segmentation of the iris. Here, interactions with eye color (and hence contrast with the pupil) were observed empirically as dark eyes yielded lower match rates. While the data set used in the present simulation was too small to draw statistically significant conclusions, similar observations for the relevance of eye color can be found in related research [27. This interplay was particularly observed for selected pathologies: 
Corneal bleaching was only accepted for subjects with very bright iris color. Further bleaching had only limited effects at the initial and intermediate stages. However, advanced corneal bleaching led to rejections in most cases as iris segmentation (in particular, the detection of the outer iris boundary) becomes problematic. Central keratitis was frequently rejected in subjects with a bright iris color, again owing to segmentation problems. Although this occurred for all subjects, the FRR was higher with bright-eyed subjects. High density infiltrates was frequently rejected in subjects with a dark iris color. This effect was is similar to corneal bleaching, but results in an uneven spread of high density infiltrates.

Table 1.

(a) Iris FRR for different pathologies

\begin{tabular}{ll} 
Pathology & Total FRR \\
\hline Corneal bleaching & $65.2 \%$ \\
Central keratitis & $61.7 \%$ \\
Change in iris color & $0.5 \%$ \\
Infiltrates, high density & $61.8 \%$ \\
Infiltrates, low density & $32.8 \%$ \\
Glaucoma surgery scar & $0.0 \%$ \\
Corneal scarring & $72.5 \%$ \\
Corneal scarring w/bleaching & $86.8 \%$ \\
Vessel growth & $6.6 \%$
\end{tabular}

(b) Retinal FRR for different pathologies

\begin{tabular}{ll} 
Pathology & Total FRR \\
\hline Blurred lens & $0.0 \%$ \\
Cotton-wool spots & $11.7 \%$ \\
Drusen & $43.3 \%$ \\
Exudates & $5.0 \%$ \\
Hemorrhage, large & $38.3 \%$ \\
Hemorrhage, small & $38.3 \%$ \\
Vessel growth & $68.3 \%$
\end{tabular}

For the retina portion, 20 subjects were chosen, and the threshold for matching was kept at a correlation coefficient of 0.7 . Tests were carried out during the adjustment phase of our algorithm, and they proved that a threshold of 0.7 would work most of the time, with a data set of images taken with visible light. Only one healthy image per retina test subject was available, the same that was used for the manipulations, which shifted results towards lower correlation coefficients (and hence FRR). Table 1b summarizes the findings for all simulations of pathologies affecting the retina. As shown in table $1 \mathrm{~b}$, neovascularization had the biggest impact on FRR among the simulated pathologies with an average FRR of $68.33 \%$. The other blood-related signs of disease, i.e. hemorrhaging, resulted in an average FRR of $38.3 \%$. The noise from these signs of disease were darker, and covered a larger area than exudates, drusen, and cotton-wool spots with higher density. The results indicate that darker eye color or illumination results in a lower FRR in general but our results do not allow the clear distinction between individual factors. This is the case for the signals acquired for the experiments discussed here (using visible-spectrum light) but must be expected to be even more pronounced in case near-infrared light is used for signal acquisition as near-IR light is absorbed effectively by blood vessels and hence also by hemorrhage artifacts. Finally, as was the case for iris recognition, we also observed a similar possible connection between retina color and illumination and the number of matches in general. The full results of the experimental study reported here can be found in 22 . 


\section{Related Work}

As noted in section 1, the extent of literature on the subject of the present paper appears to be somewhat limited. Notably, however, Roizenblatt et al. 28. performed a quantitative study on the impact of cataract surgery on the texture of affected irises and their effects on a specific iris recognition system and algorithm while Smith discusses the effects of iris pigmentation on recognition performance as well as the effects and implications of ocular illumination for signal acquisition 27. As noted in section 2, the lack of an available retina recognition system has resulted in the authors of the present paper implenting a variant of Hill's system; details on the image acquisition system used as well as on the retina recognition algorithm developed for the experiments described in this paper can be found in $22[10$.

\section{Conclusion}

While the results of the present study can only be seen as a preliminary study given the limitations imposed by the small data set and the need to resort to the simulation of pathologies which should be followed up by a longitudinal study with a sufficiently large population to allow statistical analysis over factors such as average disease progression times, the qualitative results from the simulation indicate that for application areas where biometric identification and authentication may occur only infrequently and on an irregular basis (e.g. in border control applications), disease progression can occur sufficiently rapidly to be problematic for recognition performance in aggressive forms. As an example, corneal bleaching at disease progression stages between 3 and 6 months after onset will result in a FRR of $67 \%, 78 \%$, and $87 \%$, respectively, for iris recognition (for these results, the full UBIRIS database was used with 231 subjects, resulting in a $95 \%$ confidence interval for the values given). Moreover, future work should also investigate the effect of iris color on recognition performance for both iris and retina recognition as well as other morphological features that can affect recognition performance, particularly the ability to accurately segment eye images. This in turn may yield further insights for the optimization of segmentation heuristics and hence result in improved recognition performance.

Acknowledgments. The authors would like to thank Robert "Buzz" Hill for advice and discussions on the development of retina recognition algorithms, Dr. Farshad Heybaran of the Opthalmology Department at Sykehuset Innlandet Lillehammer and Dr. Vibeke Sundling at the Department of Optometry and Visual Science at Buskerud University College for discussions on physiological properties of the ocular system and retina. Nurse Nina Løvli Wangen at Sykehuset Innlandet Lillehammer kindly assisted with the acquisition of retinal images. 


\section{References}

1. Bundesamt für Sicherheit in der Informationstechnik: Public Final Report: Evaluation of Fingerprint Recognition Technologies - BioFinger. Technical report, BSI and BKA, Bonn, Germany (August 2004)

2. Proença, H., Alexandre, L.A.: UBIRIS: A Noisy Iris Image Database. In: Roli, F., Vitulano, S. (eds.) ICIAP 2005. LNCS, vol. 3617, pp. 970-977. Springer, Heidelberg (2005)

3. Simon, C., Goldstein, I.: A New Scientific Method of Identification. New York State Journal of Medicine 35(18), 901-906 (1935)

4. Simon, C.: The Retina Method of Authentication. Series 4: Unpublished Writings by Carleton Simon, 1900-1925, 1935-1938, 1940-1945, Files A-S. M. E. Grenander Department of Special Collections \& Archives, State University of New York at Albany (1936)

5. Bolle, R., Pankanti, S., Jain, A.K.: Biometrics: Personal Identification in a Networked World. Kluwer Academic Publishers, Dordrecht (1998)

6. Woodward, J.D., Orlans, N.M., Higgins, P.T.: Biometrics: Identity Assurance in the Information Age. Osborne McGraw Hill, New York (2003)

7. Hill, R.B.: Apparatus and method for identifying individuals through their retinal vasculature pattern. U.S. Patent 4109237 (1978)

8. Hill, R.B.: Fovea-centered eye fundus scanner. U.S. Patent 4620318 (1986)

9. Samples, J.R., Hill, R.V.: Use of infrared fundus reflection for an identification device. American Journal of Ophthalmology 98(5), 636-640 (1984)

10. Borgen, H., Bours, P., Wolthusen, S.D.: Visible-Spectrum Biometric Retina Recognition. In: Proc. 4th Int'l. Conf. IIH-MSP, Harbin, China, pp. 1056-1062. IEEE Press, Los Alamitos (2008)

11. Wildes, R.P.: Iris Recognition: An Emerging Biometric Technology. Proc. IEEE 85(9), 1348-1363 (1997)

12. Flom, L., Safir, A.: Iris Recognition System. U.S. Patent 4641349 (1987)

13. Daugman, J.: How Iris Recognition Works. IEEE Transactions on Circuits and Systems for Video Technology 14(1), 21-30 (2004)

14. Daugman, J.: Probing the Uniqueness and Randomness of IrisCodes: Results From 200 Billion Iris Pair Comparisons. Proc. IEEE 94(11), 1927-1935 (2006)

15. Nanavati, S., Thieme, M., Nanavati, R.: Biometrics: Identity Verification in a Networked World. John Wiley \& Sons, New York (2002)

16. Wayman, J.L., Jain, A.K., Maltoni, D., Maio, D.: Biometric Systems: Technology, Design and Performance Evaluation. Springer-Verlag, Berlin (2005)

17. Masek, L., Kovesi, P.: MatLab Source Code for a Biometric Identification System Based on Iris Patterns. B.Sc. thesis, U. of Western Australia (May 2003)

18. Cole III, R.M.: Glaucoma. Review of Optometry 143(5) (May 2006)

19. Teus, M.A., Arranz-Márquez, E., Lucea-Suescun, P.: Incidence of iris colour change in latanoprost treated eyes. British Journal of Ophthalmology 86(10), 1085-1088 (2002)

20. Nowak, J.Z.: Age-Related Macular Degeneration (AMD): Pathogenesis and Therapy. Pharmacological Reports 58(3), 353-363 (2006)

21. Gehrs, K.M., Anderson, D.H., Johnson, L.V., Hageman, G.S.: Age-related macular degeneration - Emerging pathogenetic and therapeutic concepts. Annals of Medicine 38(7), 450-471 (2006)

22. Borgen, H.: The Effects of Eye Disease and Aging of the Eye on Biometric Authentication. Master's thesis, Gjøvik University College, Gjøvik, Norway (July 2007) 
23. Allen, D., Vasavada, A.: Cataract and surgery for cataract. British Medical Journal 333(7559), 128-132 (2006)

24. Torpy, J.M., Glass, T.J., Glass, R.M.: Retinopathy. Journal of the American Medical Association 293(1), 128 (2005)

25. Gariano, R.F., Gardner, T.W.: Retinal angiogenesis in development and disease. Nature 438(7070), 960-966 (2005)

26. Pavan-Langston, D.: Diagnosis and therapy of common eye infections: Bacterial, viral, fungal. Comprehensive Therapy 9(5), 33-42 (1983)

27. Smith, K.N.: Analysis of Pigmentation and Wavefront Coding Acquisition in Iris Recognition. Master's thesis, West Virginia U., Morgantown, WV, USA (May 2007)

28. Roizenblatt, R., Schor, P., Dante, F., Roizenblatt, J., Belfort Jr., R.: Iris recognition as a biometric method after cataract surgery. BioMedical Engineering OnLine 3(2), $1-7(2004)$ 